\title{
A BIORTHOGONAL EXPANSION RELATED TO THE ZEROS OF AN ENTIRE FUNCTION
}

\author{
HAROLD E. BENZINGER
}

ABSTRACT. Let $D(\lambda)=a_{0}+\Sigma_{k=1}^{\infty} a_{k} e^{\lambda / k}$, where the $a_{k}$ 's are given complex constants, with $a_{0} a_{1} \neq 0, \Sigma_{k=1}^{\infty}\left|a_{k}\right|<\infty$. Let $\left\{\lambda_{k}\right\}, k=1,2$, ... , denote the sequence of distinct zeros of $D(\lambda)$, labeled in order of increasing modulus, and with multiplicities $m_{k} \geq 1$. Let $\left\{\phi_{l}(x)\right\}$ denote the sequence of functions $\left\{x^{j} \exp \left(\lambda_{k} x\right): j=0, \cdots, m_{k}-1 ; k=1,2, \cdots\right.$; $0 \leq x \leq 1\}$. We show that for each $p, 1 \leq p<\infty$, there is a sequence $\left\{\psi_{l}(x)\right\}$ in $L^{q}(0,1)(p q=p+q)$ such that $\left(\phi_{l}, \psi_{m}\right)=\delta_{l m}$. Then we show that $\left\{\phi_{l}\right\}$ is complete in $L^{p}(0,1), 1 \leq p<\infty$, and for $1<p<\infty$, we find a subspace of $L^{p}(0,1)$ such that the biorthogonal expansion $f=\Sigma_{k=1}^{\infty}\left(f, \psi_{k}\right) \phi_{k}$ is valid in the norm of $L^{p}(0,1)$.

1. Introduction. For fixed $p, 1 \leq p \leq \infty$, let $T=T(p)$ denote the linear operator taking $L^{p}(0,1)$ into $L^{p}(0,1)$ defined by $T u=u^{(1)}$, where the domain $D=D(T(p))$ consists of all absolutely continuous members $u$ of $L^{p}(0,1)$ such that $u^{(1)} \in L^{p}(0,1)$, and such that

$$
a_{0} u(0)+\sum_{k=1}^{\infty} a_{k} u(1 / k)=0,
$$

when the $a_{k}$ 's are as given above. Then it is easily verified that the eigenvalues of $T$ are the zeros of $D(\lambda)$, the eigenfunctions are of the form $\exp \left(\lambda_{k} x\right)$, and the generalized eigenfunctions are of the form $x^{j} \exp \left(\lambda_{k} x\right)$. For $1 \leq p<\infty, D(T(p))$ is dense in $L^{p}(0,1)$, so an adjoint operator $T^{*}$ : $L^{q}(0,1) \rightarrow L^{q}(0,1)$ exists. The sequence $\left\{\psi_{l}\right\}$ thus consists of the eigenfunctions and generalized eigenfunctions of $T^{*}$. The proofs of the statements concerning completeness and the behavior of the biorthogonal expansions thus depend upon obtaining suitable estimates for the resolvent operator $R(\lambda, T)$ of $T$ for large $|\lambda|$.

Since $|D(\lambda)| \leq K e^{|\lambda|}$ for some constant $K$, we see that $D(\lambda)$ is an example of an entire function of exponential type [3, p. 84]. In addition, the

Presented to the Society, April 27, 1973; received by the editors March 12, 1973 and, in revised form, February 15, 1974. 34B 10.

AMS (MOS) subject classifications (1970). Primary 35P10; Secondary 30A18, 
condition $a_{0} a_{1} \neq 0$ implies that

$$
\lim _{x \rightarrow-\infty}|D(x)|>0, \quad \lim _{x \rightarrow+\infty}|D(x)| e^{-x}>0 .
$$

Thus by suitably modifying the statement of Theorem (d) in [3, p. 418] to take into account the change of interval and the possibility of multiple zeros, we see that the completeness of the system $\left\{\phi_{k}\right\}$ in $L^{p}(0,1), 1 \leq p<\infty$, can be proved without reference to differential operators.

The advantage in our approach is that we obtain the additional information concerning the behavior of the biorthogonal expansion. In the course of the discussion, we shall see that the partial sums of the biorthogonal expansion can be related to the partial sums of the usual Fourier series expansion. Furthermore, the operator $T$ has an infinite number of boundary values, so that our method is of interest as an approach to the spectral theory of such operators.

Ordinary differential operators with infinitely many boundary values have been discussed in [4] and [5]. The idea of using boundary conditions such that the corresponding $D(\lambda)$ has as frequencies the infimum and supremum of its frequencies can be found in [4]. The eigenfunction expansion theorem in [4], while appearing plausible, is based on Lemmas 4.2 and 4.3 of [4], which appear to be false.

2. The adjoint and the resolvent of $T$. Since $u$ is continuous and $u^{(1)} \in L^{p}(0,1)$, we see that $u(x)=u(1)-\int_{x}^{1} f(t) d t$, where $f \in L^{p}(0,1)$. To impose the condition (1.1), introduce the notation

$$
A=\sum_{k=0}^{\infty} a_{k} ; \quad H(x)=\bar{a}_{0}+\sum_{l=k+1}^{\infty} \bar{a}_{l} \quad \text { for } \quad 1 /(k+1)<x<1 / k .
$$

Then we see that $u \in D(T)$ if and only if

$$
u(x)=u(1)-\int_{x}^{1} f(t) d t, \quad u(1) A=\int_{0}^{1} f(t) \bar{H}(t) d t, \quad f \in L^{p}(0,1) .
$$

Using (2.1), we see that the adjoint relationship $(T u, v)=(u, w)$ holds for all $u \in D(T)$ and some $v, w$ in $L^{q}(0,1)$ if and only if

$$
v(x)=-\int_{0}^{x} w(t) d t+\alpha H(x), \quad \bar{A} \alpha=\int_{0}^{1} w(x) d x, \quad w \in L^{q}(0,1) .
$$

This characterizes $T^{*}$. Since $\int_{0}^{x} w(t) d t$ is continuous, we see that $v(x)$ has jump discontinuities at the points $x=1 / k, k=2,3, \cdots$. Thus to solve the equation $T^{*} v=\lambda v$, we consider all functions of the form 


$$
v(x)=e^{-\lambda x} \sum_{k=1}^{\infty} \delta_{k} \chi_{k}(x),
$$

where $\chi_{k}$ is the characteristic function of the interval $I_{k}=(1 /(k+1), 1 / k)$, and the $\delta_{k}$ 's are selected so that $v \in L^{q}(0,1)$. We then seek to determine those values of $\lambda$ for which the $\delta_{k}$ 's can also be selected so that $v$ satisfies (2.2). Straightforward computation shows that we must have $\bar{D}(\lambda)=0$, so the eigenvalues $\nu_{k}$ of $T^{*}$ satisfy $\nu_{k}=\bar{\lambda}_{k}$. In addition, the algebraic and geometric multiplicities for $\lambda_{k}$ and $\nu_{k}$ coincide. Consequently, if $\left\{\Phi_{j k}: 0 \leq j \leq m_{k}-1\right\}$ and $\left\{\Psi_{j k}: 0 \leq j \leq m_{k}-1\right\}$ denote the eigenfunctions and generalized eigenfunctions of $T$ and $T^{*}$, respectively, at $\lambda_{k}$ and $\nu_{k}$, then we have

$$
\left(\Phi_{j k}, \Psi_{l r}\right)=\delta_{k r} \delta_{j m_{k}-j}
$$

If we let $\left\{\psi_{l}\right\}$ denote the sequence of functions $\Psi_{j k}, k=1,2, \cdots$, then with appropriate labeling, $\left(\phi_{k}, \psi_{j}\right)=\delta_{k j}$.

To find an expression for the resolvent of $T$, we note that for fixed $f \in L^{p}(0,1)$, the differential equation $u^{(1)}=\lambda u+f$ has absolutely continuous solutions of the form

$$
\begin{gathered}
u(x, \lambda)=c e^{\lambda x}+e^{\lambda x} \int_{0}^{x} e^{-\lambda t} f(t) d t \\
u(x, \lambda)=d e^{\lambda x}-e^{\lambda x} \int_{x}^{1} e^{-\lambda t} f(t) d t
\end{gathered}
$$

If $\lambda$ is not an eigenvalue of $T$, so that $D(\lambda) \neq 0$, then $c, d$ can be uniquely determined so that $u \in D(T)$. Let

$$
D_{k}(\lambda)=\sum_{l=1}^{k} a_{l} e^{\lambda / l}, \quad R_{k}(\lambda)=a_{0}+\sum_{l=k+1}^{\infty} a_{l} e^{\lambda / l} .
$$

Let $\int_{k} F$ denote the integral of $F$ over the interval $I_{k}$. Then

$$
\begin{aligned}
& u(x, \lambda)=-D^{-1}(\lambda) \sum_{k=1}^{\infty} \int_{k} D_{k}(\lambda) e^{\lambda(x-t)} f(t) d t+\int_{0}^{x} e^{\lambda(x-t)} f(t) d t \\
& u(x, \lambda)=D^{-1}(\lambda) \sum_{k=1}^{\infty} \int_{k} R_{k}(\lambda) e^{\lambda(x-t)} f(t) d t-\int_{x}^{1} e^{\lambda(x-t)} f(t) d t
\end{aligned}
$$

Consequently, 


$$
u(x, \lambda)=-R(\lambda, T) f=\int_{0}^{1} G(x, t, \lambda) f(t) d t,
$$

where the Green's function $G$ of $T$ is given by

$$
\begin{array}{cc}
G(x, t, \lambda)=D^{-1}(\lambda) R_{k}(\lambda) e^{\lambda(x-t)}, & 0<t<x<1, t \in I_{k}, \\
G(x, t, \lambda)=D^{-1}(\lambda) D_{k}(\lambda) e^{\lambda(x-t)}, & 0<x<t<1, t \in I_{k}
\end{array}
$$

It is also useful to define

$$
\begin{gathered}
\Gamma(x, t, \lambda)=e^{\lambda(x-t)}, \quad 0<t<x<1, R \lambda \leq 0, \\
\Gamma(x, t, \lambda)=0, \quad 0<x<t<1, R \lambda \leq 0, \\
\Gamma(x, t, \lambda)=0, \quad 0<t<x<1, R \lambda \geq 0, \\
\Gamma(x, t, \lambda)=-e^{\lambda(x-t)}, \quad 0<x<t<1, \mathbb{R} \lambda \geq 0 .
\end{gathered}
$$

Then from (2.8) and (2.9), we see that

$$
\int_{0}^{1}[G(x, t, \lambda)-\Gamma(x, t, \lambda)] f(t) d t
$$

$$
\begin{gathered}
=-D^{-1}(\lambda) \sum_{k=1}^{\infty} \int_{k} D_{k}(\lambda) e^{\lambda(x-t)} f(t) d t \quad(\Re \lambda \leq 0) \\
=D^{-1}(\lambda) \sum_{k=1}^{\infty} \int_{k} R_{k}(\lambda) e^{\lambda(x-t)} f(t) d t \quad(\Re \lambda \geq 0) .
\end{gathered}
$$

Let $C_{R}$ denote the contour $|\lambda|=R$. Note that

$$
-\frac{1}{2 \pi i} \int_{C_{R}} \Gamma(x, t, \lambda) d \lambda=\frac{\sin R(x-t)}{\pi(x-t)},
$$

the Dirichlet kernel for the Fourier transform.

3. The completeness and expansion theorems. The following theorem is a summary of results which can be found in [3, Chapter VI, \$2].

Theorem 3.1. The zeros $\left\{\lambda_{k}\right\}$ of $D(\lambda)$ lie in a strip $|R \lambda| \leq h$ for some $h>0$. The number of zeros of $D(\lambda)$ in any rectangle $|\Re \lambda| \leq h, t<\mathfrak{T} \lambda \leq t+$ 1 , is bounded by a constant not depending on $t$. If $\delta>0$ is given, then

$$
\begin{aligned}
\left|D^{-1}(\lambda)\right| \leq K\left|e^{-\lambda}\right|, \quad & R \lambda \geq 0, \quad\left|\lambda-\lambda_{k}\right| \geq \delta, \\
\left|D^{-1}(\lambda)\right| \leq K, \quad & R \lambda \leq 0, \quad\left|\lambda-\lambda_{k}\right| \geq \delta .
\end{aligned}
$$


Note that estimates (3.1), (3.2) use the condition $a_{0} a_{1} \neq 0$. In addition, since the sequence $\left\{\lambda_{k}\right\}$ is not too dense, for each $r>0$ sufficiently large, the annulus $2 r \leq|\lambda| \leq 3 r$ contains a circle $C_{R}$ such that $\left|\lambda-\lambda_{k}\right| \geq \delta$ for all $\lambda_{k}$ when $\lambda$ is on $C_{R}$, provided $\delta>0$ is sufficiently small.

Theorem 3.2. The eigenfunctions of $T$ are complete in $L^{p}(0,1), 1 \leq p$ $<\infty$.

Proof. We show that $R(\lambda, T)$ satisfies the hypothesis of Theorem 1.1 in [2]. The assumption, there, that $R(\lambda, T)$ is a compact operator is merely a convenience to insure that the residue of $R(\lambda, T)$ at $\lambda_{k}$ has the form given in [2, equation (1.1)]. In the present case, we have verified directly in $\$ 2$ that the projections $P_{k}$ have the required form. In fact, for $p=1$, it is unlikely that $R(\lambda, T)$ is compact.

Thus it remains to show that $\|R(\lambda, T)\|_{p} \leq K_{p}|\lambda|^{\mu}$ for some $\mu$ and sufficiently large $|\lambda| \neq\left|\lambda_{k}\right|$. Using (2.7), we see that if $\lambda$ is on $C_{R},\left|\lambda-\lambda_{k}\right| \geq$ $\delta$, and $R \lambda \geq 0$, then

$$
\left|D^{-1}(\lambda) R_{k}(\lambda)\right| \leq K \mid \exp \left\{\lambda\left((1 /(k+1))^{-1}\right\}|,| D^{-1}(\lambda) D_{k}(\lambda) \mid \leq K,\right.
$$

while for $R \lambda \leq 0$,

$$
\left|D^{-1}(\lambda) R_{k}(\lambda)\right| \leq K, \quad\left|D^{-1}(\lambda) D_{k}(\lambda)\right| \leq K\left|e^{\lambda / k}\right| .
$$

$K$ stands for an absolute constant, not necessarily the same on each occurrence. For fixed $t \in I_{k}$, we obtain from (2.11), (2.12), (3.3), (3.4), for $R \lambda \geq 0$,

$$
\begin{aligned}
& |G(x, t, \lambda)| \leq K \exp \{\Re \lambda[x-1-t+1 / k+1]\} \leq K, \quad t<x, \\
& |G(x, t, \lambda)| \leq K \exp \{\Re \lambda(x-t)\} \leq K, \quad x<t,
\end{aligned}
$$

while for $R \lambda \leq 0$,

$$
\begin{aligned}
& |G(x, t, \lambda)| \leq K \exp \{\Re \lambda(x-t)\} \leq K, \quad t<x, \\
& |G(x, t, \lambda)| \leq K \exp \{\Re \lambda(x-t+1 / k)\} \leq K, \quad x<t .
\end{aligned}
$$

Thus $|G(x, t, \lambda)| \leq K$, uniformly in $x, t$, and $R$, provided $\left|\lambda-\lambda_{k}\right| \geq \delta$. Thus for each $p, 1 \leq p<\infty,\|R(\lambda, T)\|_{p} \leq K$.

To discuss the convergence of the biorthogonal expansion of $f$, let

$$
\begin{gathered}
K(x, t, R)=\frac{1}{2 \pi i} \int_{C_{R}}[G(x, t, \lambda)-\Gamma(x, t, \lambda)] d \lambda, \\
\left(J_{R} f\right)(x)=\int_{0}^{1} K(x, t, R) f(t) d t .
\end{gathered}
$$

Then using (2.10), we have 


$$
\frac{1}{2 \pi i} \int_{C_{R}} R(\lambda, T) f d \lambda=\int_{-R}^{R} \frac{\sin R(x-t)}{\pi(x-t)} f(t) d t-\left(J_{R} f\right)(x) .
$$

Since the first term on the right side of (3.7) converges to $f$ in the norm of $L^{p}(0,1), 1<\dot{p}<\infty$, the biorthogonal expansion will be valid for those $f$ such that $J_{R} f$ converges to zero as $R . \rightarrow \infty$.

Lemma 3.1. For $R>0$ sufficiently large,

$$
|K(x, t, R)| \leq M\left\{[1-x+t-1 / k+1]^{-1}+[x-t+1 / k]^{-1}\right\},
$$

$$
0<x<1, t \in I_{k} \text {. }
$$

Proof. Let $C_{R}^{+}$denote that portion of $C_{R}$ in the right half-plane, and similarly for $C_{R}^{-}$. Then

$$
|K(x, t, R)| \leq M\left[\int_{C_{R}^{+}}|G-\Gamma||d \lambda|+\int_{C_{R}^{-}}|G-\Gamma||d \lambda|\right] .
$$

Since, from (2.17) and (2.18),

$$
\begin{aligned}
G(x, t, \lambda)-\Gamma(x, t, \lambda) & =-D^{-1}(\lambda) D_{k}(\lambda) e^{\lambda(x-t)} & & (\Re \lambda \leq 0) \\
& =D^{-1}(\lambda) R_{k}(\lambda) e^{\lambda(x-t)} & & (R \lambda \geq 0),
\end{aligned}
$$

we see that using the estimates (3.3), (3.4), for $t \in I_{k}$ and $0,<x<1$,

$$
|G(x, t, \lambda)-\Gamma(x, t, \lambda)| \leq M \exp R(x+1 / k-t) \cos \theta,
$$

$$
\begin{array}{r}
\pi / 2 \leq \theta \leq 3 \pi / 2, \\
|G(x, t, \lambda)-\Gamma(x, t, \lambda)| \leq M \exp R(x-1-t+1 / k+1) \cos \theta, \\
-\pi / 2 \leq \theta \leq \pi / 2 .
\end{array}
$$

Using the inequality $\cos \theta \geq 2 \theta / \pi+1$ for $-\pi / 2 \leq \theta \leq 0$, similar inequalities for the other values of $\theta$, and using $|d \lambda|=R d \theta$, we can make the appropriate substitutions in (3.10) and (3.11), and then integrate directly, obtaining (3.8).

Lemma 3.2. Let $f \in C^{1}[0,1]$ satisfy $f(1 / k)=0$, all $k$, and assume $f(x)=0$ if $0 \leq x \leq 1 / L$ for some integer $L$. Then

$$
\left|\left(J_{R} f\right)(x)\right| \leq \frac{M}{R} \sum_{k=1}^{L-1} \int_{k} H_{k}(x, t)\left|f^{(1)}(t)\right| d t,
$$

where $H_{k}$ is the right side of (3.8).

Proof. Since $\int_{k} e^{-\lambda t} f(t) d t=(1 / \lambda) \int_{k} e^{-\lambda t} f(1)(t) d t$, we see that 


$$
\left(J_{R} f\right)(x)=\int_{0}^{1} K_{1}(x, t, R) f^{(1)}(t) d t
$$

where

$$
K_{1}(x, t, R)=\frac{1}{2 \pi i} \int_{C_{R}} \frac{1}{\lambda}[G(x, t, \lambda)-\Gamma(x, t, \lambda)] d \lambda .
$$

We then repeat the estimates from Lemma 3.1, starting with a factor of $1 / R$.

For $g \in L^{p}(0,1)$, let $\left\|g_{k}\right\|_{p}^{p}=\int_{k}|g(t)|^{p} d t$, and for $h \in L^{\infty}(0,1)$, let $\left\|h_{k}\right\|_{\infty}=$ ess $\sup \left\{|h(x)|: x \in I_{k}\right\}$.

Theorem 3.3. If $f \in L^{p}(0,1), 1<p<\infty$, if $f=h g$, where $g \in L^{p}(0,1)$, $h \in L^{\infty}(0,1)$, and if $\left\{\left\|h_{k}\right\|_{\infty}\right\}$ is in $l^{q}(p q=p+q)$, then there exists a constant $K_{p}(h)>0$ such that $\left\|J_{R} f\right\|_{p} \leq K_{p}(h)\|g\|_{p}, R \rightarrow \infty$.

Proof. Let $F_{k}(x)=\int_{k} H_{k}(x, t)|f(t)| d t, 0<x<1$. Then using Lemma 3.1, we have

$$
\left|\left(J_{R} f\right)(x)\right| \leq M \sum_{k=1}^{\infty}\left|F_{k}(x)\right|
$$

Since the Hilbert transform is a bounded linear transformation of $L^{p}(0,1) \rightarrow$ $L^{p}(0,1)$ for $1<p<\infty$, we see that

$$
\left[\int_{0}^{1}\left|F_{k}(t)\right|^{p} d t\right]^{1 / p} \leq H_{p}\left\|f_{k}\right\|_{p} \leq H_{p}\left\|h_{k}\right\|_{\infty}\left\|g_{k}\right\|_{p} .
$$

Since $\left\{\left\|h_{k}\right\|_{\infty}\right\}$ is in $l^{q}$ and $\left\{\left\|g_{k}\right\|_{p}\right\}$ is in $l^{p}$, we have

$$
\left\|J_{R} f\right\|_{p} \leq H_{p}\left(\sum_{1}^{\infty}\left\|h_{k}\right\|_{\infty}^{q}\right)^{1 / q}\left(\sum_{1}^{\infty}\left\|g_{k}\right\|_{p}^{p}\right)^{1 / p}=K_{p}(h)\|g\|_{p} .
$$

Theorem 3.4. If $f$ satisfies the hypothesis of Lemma 3.2, then $\lim _{R \rightarrow \infty}\left\|J_{R} f\right\|_{p}=0,1<p<\infty$.

Proof. Since $f^{(1)}$ is zero for $x<1 / L$, let $h$ be the characteristic function of $[1 / L, 1]$. Then using (3.12),

$$
\left\|J_{R} f\right\|_{p} \leq(1 / R) K_{p}(h)\left\|f^{(1)}\right\|_{p}
$$

Theorem 3.5. If $f$ satisfies the bypotheses of Theorem 3.3, then

$$
\left\|f-\frac{1}{2 \pi i} \int_{C_{R}} R(\lambda, T) f d \lambda\right\|_{p} \rightarrow 0 \text { as } R \rightarrow \infty .
$$

Proof. By Theorem 3.3, the linear operator $g \rightarrow J_{R}(h g)$ is uniformly bounded on $L^{p}(0,1)$, and by Theorem 3.4, it converges to zero on a dense 
subspace of $L^{p}(0,1)$. Thus $J_{R}(h g)$ converges to zero for all $g$ in $L^{p}(0,1)$. The result follows then from equation (3.7).

\section{REFERENCES}

1. H. E. Benzinger, The $L^{p}$ behavior of eigenfunction expansions, Trans. Amer. Math. Soc. 174 (1972), 333-344.

2. - Completeness of eigenvectors in Banach spaces, Proc. Amer. Math. Soc. 38 (1973), 319-324.

3. B. Ja. Levin, Distribution of zeros of entire functions, GITTL, Moscow, 1956; English transl., Transl. Math. Monographs, vol. 5, Amer. Math. Soc., Providence, R. I., 1964. MR 19, 402; 28 \#217.

4. A. M. Krall, An eigenfunction expansion for a nonselfadjoint, interior point boundary value problem, Trans. Amer. Math. Soc. 170 (1972), 137-147. MR 47 \#547.

5. R. C. Brown, G. B. Green and A. M. Krall, Eigenfunction expansions under multipoint-integral boundary conditions, Ann. Mat. Pura Appl. 95 (1973), 231-243.

DEP ARTMENT OF MATHEMATICS, UNIVERSITY OF ILLINOIS, URBANA, ILLINOIS 61801 